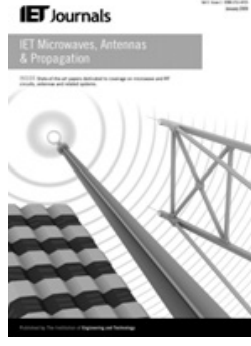

\title{
Shunt series LC circuit for compact coplanar waveguide notch filter design
}

\author{
Juan de Dios Ruiz, Juan Hinojosa \\ Department of Electronics and Computer Engineering, Universidad Politécnica de Cartagena, Plaza del Hospital no 1, \\ 30202 Cartagena, Murcia, Spain \\ E-mail: juan.hinojosa@upct.es
}

\begin{abstract}
A shunt series $L C$ resonant circuit based on an open version of an interconnected split ring resonator (OISRR) is proposed. This OISRR exhibits half the resonant frequency of the split ring resonator, allows a parallel connection with a coplanar waveguide (CPW) section and according to its frequency response is an attractive cell for compact CPW notch filter design. $3 \mathrm{~dB}$ stop-band bandwidth lower than $4.5 \%$ with more than $11 \mathrm{~dB}$ insertion loss in the stop-band can be achieved with a CPW loaded with a single OISRR. The experimental results have confirmed the possibilities of this OISRR, which can be easily connected to CPW antennas to reject undesirable signals.
\end{abstract}

\section{Introduction}

The recent developments and challenges in communication systems focus on designing and producing compact microwave circuits at low cost with a higher communication capacity. Filters, planar technology and metamaterialinspired structures are essential in the design of these modern communication systems. The filters are important devices to reject unwanted signals. Planar filters are popular and have low cost and light weight. In addition, such filters are easily fabricated using conventional printed circuit technology. However, planar filters require a change in geometry for the design of compact circuits [1]. In this sense, metamaterial-inspired structures are of interest for size reduction purposes [2].

Band-stop filters (BSFs) have not been extensively investigated in comparison with their band-pass and low-pass counterparts. BSFs have applications, for example in oscillators, mixers and antennas, to reject higher-order harmonics and other undesirable spurious signals. The conventional methods use shunt stubs or stepped-impedance microstrip lines to implement BSFs [1]. These filters have usually a narrow stop-band response and a large circuit size. To increase the stop-band bandwidth, it is possible to use defected ground structures and electromagnetic bandgap structures [3-5]. However, the size of these filters can be relatively large at certain frequencies, since several cells of periodic structure are needed to provide a significant level of stop-band rejection. To reduce filter size, slow-wave open-loop resonator structures and metamaterial-inspired structures have been adopted in coplanar waveguide (CPW) technology [6-9]. In these implementations, the resonators are electrically or magnetically coupled to a host CPW or connected either in series or in shunt configurations. The metamaterial-inspired structures are based on split ring resonators (SRRs), open SRRs (OSRRs) and their respective complementary structures [10-12].

In this paper, an open version of an interconnected SRR (OISRR) is proposed and analysed for novel compact design of CPW BSFs (Fig. 1). The basic OISRR cell allows a parallel connection with a CPW section and behaves as a shunt series RLC circuit. Moreover, this OISRR cell presents half the electrical size of the SRR cell. With respect to other existing alternatives in CPW technology [69], the proposed CPW OISRR-based structure is suitable for compact design, presents a narrow stop-band response, does not require tuning small gaps and does not have important geometric constraints as the slot-type or strip-type SRRs, since the rings of the OISRR are connected in parallel at a common point of the CPW. Its usefulness for compact CPW notch filters is experimentally demonstrated.

\section{Structure, equivalent circuit model and analysis of a CPW loaded with OISRRs}

Fig. 1 shows the structure and equivalent circuit model of a CPW loaded with an OISRR cell. The OISRR cell is etched in an open window $D_{1} \times D_{2}$ located in one of the two lateral ground planes of the CPW. In order to avoid the excitation of parasitic modes, the two lateral ground planes of the structure are connected by means of four via holes and four metallic strips printed in the bottom substrate layer. The basic OISRR cell is conceived from two open rings of different radii. On the upper substrate layer, the dimensions of the proposed OISRR are the radius $r$ of the external ring, the width $c$ of the ring conductors and the separation slot $s$ between rings. As long as the size of the OISRR is electrically small, the structure can be described by means of lumped elements. The OISRR is similar to the OSRR structure $[8,12]$ with the difference that the two 


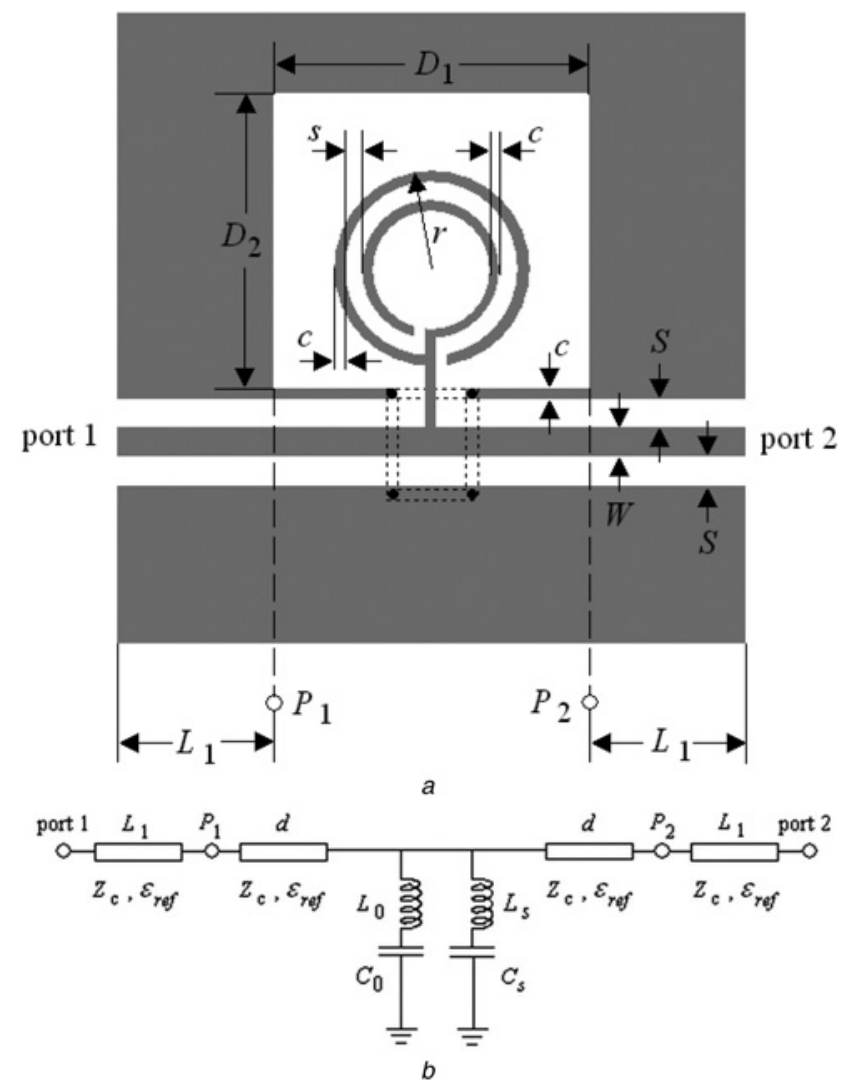

Fig. $1 C P W$ loaded with an OISRR

$a$ Top view

$b$ Equivalent circuit with two shunt series $L C$ resonant elements

rings are connected to a common point of the CPW and, therefore, instead of having a series connection, the OISRR has a parallel connection with the CPW. Thus, one can expect that the two structures (OISRR and OSRR) have the same main resonant frequency. Therefore the proposed OISRR cell can be of great interest for compact designs, since it will allow smaller electrical circuit sizes compared with the OSRR cell [12].

Fig. $1 b$ represents the equivalent circuit model for a CPW loaded with an OISRR cell. Neglecting losses, the equivalent model of the OISRR cell consists of two shunt series $L C$ resonant circuits embedded between two CPW sections of effective length $d$, which present an effective permittivity $\varepsilon_{\text {ref }}$ and a characteristic impedance $Z_{\mathrm{c}}$. Port 1 and port 2 are the input and output ports of the structure. Two frequencies $f_{0}$ and $f_{\mathrm{s}}$ can be defined from the model. $f_{0}$ and $f_{\mathrm{s}}$ correspond, respectively, to the main resonant frequency and the spurious resonance of the OISRR cell. $f_{0}$ is obtained by the resonant condition of the shunt series $L_{0} C_{0}$ circuit, which leads to a $S_{21}$ transmission zero. In the same way, $f_{\mathrm{s}}$ also leads to a $S_{21}$ transmission zero and its value is determined by means of the shunt series $L_{\mathrm{s}} C_{\mathrm{s}}$ circuit.

The element values of the shunt series $L_{0} C_{0}$ circuit (Fig. 1b) are calculated from the element transformation between the OSRR and OISRR circuit models by using an impedance scaling factor $\gamma[1,12]$

$$
\begin{gathered}
\gamma=\frac{1}{4}\left(\frac{\Delta f_{\text {OSRR }}}{\Delta f_{0}}\right) \\
L_{0}=\gamma L_{\text {OSRR }}
\end{gathered}
$$

$$
C_{0}=C_{\text {OSRR }} / \gamma
$$

where $\Delta f_{\text {OSRR }}$ and $\Delta f_{0}$ are the $3 \mathrm{~dB}$ band-pass and stop-band bandwidth at the main resonant frequencies of the OSRR and OISRR, respectively. $L_{\mathrm{OSRR}}$ is the inductance of a closed ring of average radius $r_{0}=r-c-s / 2$ and width $c$. $C_{\text {OSRR }}$ is the distributed capacitance between the inner and outer rings: $C_{\mathrm{OSRR}}=2 \pi r_{0} C_{\text {pul }}\left(C_{\mathrm{pul}}\right.$ is the capacitance per unit length between the rings). $L_{\mathrm{OSRR}}$ and $C_{\mathrm{OSRR}}$ correspond to the elements of the OSRR circuit model [12] with substrate and dimensions identical to the OISRR cell (Fig. 1a).

The shunt series $L_{\mathrm{s}} C_{\mathrm{s}}$ circuit has a reactance slope $X_{\mathrm{s}}=\omega_{\mathrm{s}} L_{\mathrm{s}}$ and resonates at $f_{\mathrm{s}}=1 /\left(2 \pi \sqrt{L_{\mathrm{s}} C_{\mathrm{s}}}\right)$. The values of the shunt series $L_{\mathrm{s}} C_{\mathrm{s}}$ circuit (Fig. $1 b$ ) are derived at the frequency $f_{\mathrm{s}}$ from the transmission parameter of the two-port network terminated with $50 \Omega$ (Fig. 1) and neglecting the effect of the shunt series $L_{0} C_{0}$ circuit as follows [1]

$$
\begin{gathered}
\frac{X_{s}}{50}=\frac{f_{s}}{2 \Delta f_{s}} \Rightarrow L_{s}=\frac{50}{4 \pi \Delta f_{s}} \\
C_{s}=\frac{1}{L_{s}}\left(\frac{1}{2 \pi f_{s}}\right)^{2}
\end{gathered}
$$

where $\Delta f_{\mathrm{s}}$ is the $3 \mathrm{~dB}$ stop-band bandwidth at $f_{\mathrm{s}}$. Finally, the effective length $d$ of the CPWs (Fig. 1) is obtained by adjusting the phase of the $S$-parameters.

Typical electromagnetic (EM) simulation and equivalent circuit results of the CPW loaded with an OISRR are shown in Fig. 2. The frequency responses were obtained
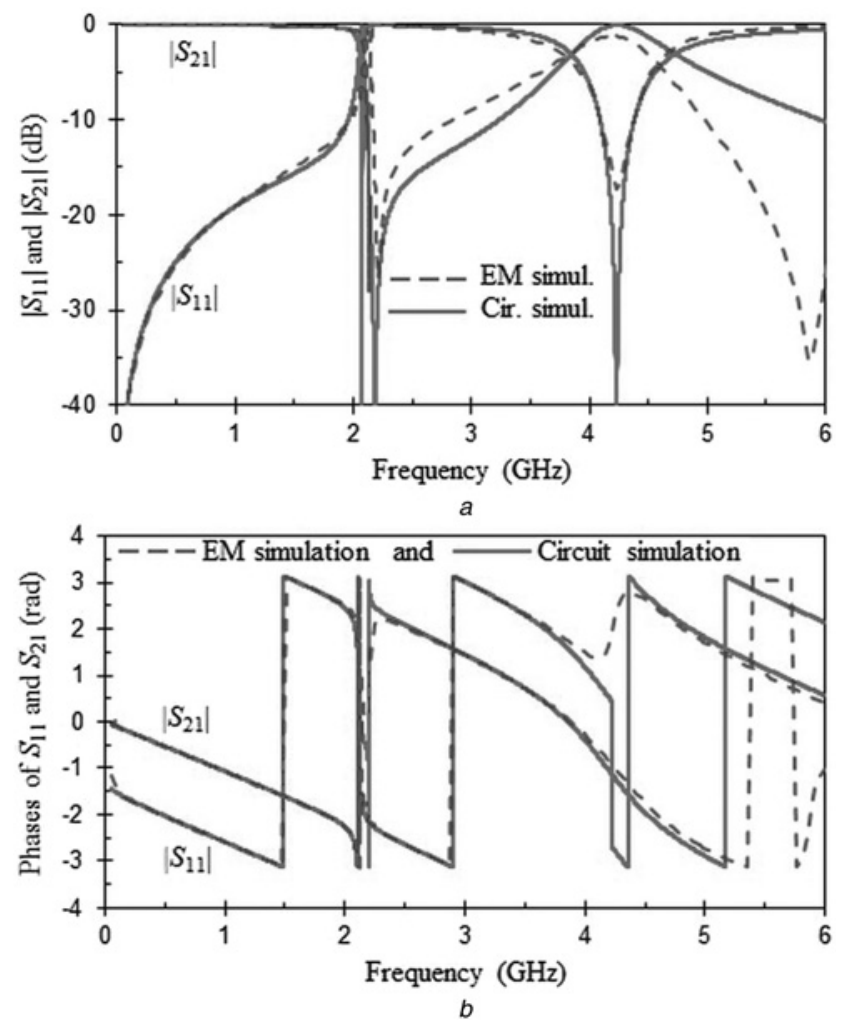

Fig. 2 EM simulation and equivalent circuit results for the $C P W$ loaded with an OISRR

OISRR data: $r=2.2 \mathrm{~mm}, c=0.3 \mathrm{~mm}, s=0.25 \mathrm{~mm}$ and $D_{1} \times D_{2}=9 \times 6.5 \mathrm{~mm}^{2}$ $a$ Magnitude

$b$ Phase 
from commercial simulators (ADS and HFSS). All the structures considered in this paper have a copper thickness $t=17.5 \mu \mathrm{m}$ and a substrate thickness $h=0.635 \mathrm{~mm}$ with a permittivity $\varepsilon_{r}=10.2$. The physical parameters of all CPWs are: $L_{1}=5.5 \mathrm{~mm}, W=0.374 \mathrm{~mm}$ and $S=0.163 \mathrm{~mm}$ for a characteristic impedance $Z_{\mathrm{c}}=50 \Omega$. The dimensions of the OISRR are: $r=2.2 \mathrm{~m}, c=0.3 \mathrm{~mm}, s=0.25 \mathrm{~mm}$ and $D_{1} \times$ $D_{2}=9 \times 6.5 \mathrm{~mm}^{2}$. The element values of the shunt series $L C$ circuits are obtained from the previous OISRR dimensions, as well as from the EM simulation results (dashed lines in Fig. 2) and the values of the OSRR model [12]: $\quad \Delta f_{0}=0.044 \mathrm{GHz}, \quad f_{\mathrm{s}}=4.23 \mathrm{GHz}, \quad \Delta f_{\mathrm{s}}=0.93 \mathrm{GHz}$ $L_{\mathrm{OSRR}}=7.4 \mathrm{nH}, \quad C_{\mathrm{OSRR}}=0.8 \mathrm{pF}$ and $\Delta f_{\mathrm{OSRR}}=2.15 \mathrm{GHz}$. The equivalent circuit parameters of the OISRR (Fig. 1b) are obtained by substituting the previous data into (1)-(5) and adjusting the phase of the $S$-parameters, giving the following values: $\gamma=12.5, L_{0}=92.5 \mathrm{nH}, C_{0}=0.064 \mathrm{pF}, L_{\mathrm{s}}$ $=4.29 \mathrm{nH}, C_{\mathrm{s}}=0.33 \mathrm{pF}$ and $d=5.1 \mathrm{~mm}$. In Fig. 2, a good agreement up to approximately $4.5 \mathrm{GHz}$ is found between the responses of the circuit model (solid lines) and EM simulation (dashed lines). The CPW OISRR-based structure has a main resonant frequency (first transmission zero) at $f_{0}$ $=2.13 \mathrm{GHz}$, an anti-resonance at $f_{\mathrm{a}}=2.2 \mathrm{GHz}$ and a spurious resonance at $f_{\mathrm{s}}=4.23 \mathrm{GHz}$, which is around two times $f_{0} \quad\left(f_{\mathrm{s}} \simeq 2 f_{0}\right)$, and presents a $3 \mathrm{~dB}$ stop-band bandwidth $\Delta f_{\mathrm{s}}=0.93 \mathrm{GHz}$ approximately 21 times greater than the main $3 \mathrm{~dB}$ stop-band bandwidth $\Delta f_{0}=0.044 \mathrm{GHz}$. The anti-resonance in the response of the CPW OISRR-based structure is because of the anti-resonant behaviour of the effective permittivity [13]. At $f_{\mathrm{a}}=2.2 \mathrm{GHz}$, the CPW OISRR-based structure behaves as an equivalent shunt parallel $L C$ resonant circuit. On the other hand, the transmission zeros occur at the resonant frequencies of the OISRR, since at these frequencies there is an electric short to ground, and the injected power is reflected back to the source. Note that at the main resonant frequency $f_{0}=2.13$ $\mathrm{GHz}$ the OISRR works as a notch filter and its resonant frequency is half that of the SRR with identical substrate and dimensions. The wavelength in the $\mathrm{CPW}$ is around $\lambda=$ $62 \mathrm{~mm}\left(\varepsilon_{\mathrm{ref}}=5.1, f_{0}=2.13 \mathrm{GHz}\right)$ and the length $D_{1}=9 \mathrm{~mm}$ of the notch filter is below $\lambda / 6$, thus confirming the small electrical size of this proposed OISRR, as compared with a traditional CPW resonator which requires a length of $\lambda / 2$.

EM and circuit simulations have been performed to study the effects of the design parameters $r, c$ and $s$ of the proposed notch filter on the main resonant frequency $\left(f_{0}\right)$ and the $3 \mathrm{~dB}$ stop-band bandwidth $\left(\Delta f_{0}\right)$. The results are presented in Fig. 3. This study will also serve to validate the equivalent circuit model. The circuit and EM simulation results have shown that the anti-resonance $f_{\mathrm{a}}$ and the spurious resonance $f_{\mathrm{s}}$ appear, respectively, above the main resonance $f_{0}$ and approximately around two times the frequency $f_{0}\left(f_{\mathrm{s}} \simeq 2 f_{0}\right)$. In Fig. 3 , the dashed lines show the results of the EM simulations, while the solid lines indicate the results obtained from the equivalent circuit. In Fig. $3 a$ ( $c=0.3 \mathrm{~mm}$ and $s=0.25 \mathrm{~mm}$ fixed), it can be seen that the main resonant frequency $\left(f_{0}\right)$ and the $3 \mathrm{~dB}$ stop-band bandwidth $\left(\Delta f_{0}\right)$ decrease as the radius $r$ of the external ring increases from 2 to $2.4 \mathrm{~mm}$. This is because the values of the capacitance $C_{0}$, the inductance $L_{0}$ and the reactance slope $X_{0}\left(X_{0}=\omega_{0} L_{0}\right)$ of the OISRR circuit model increase as the radius $r$ is incremented. This trend is reverted when either the width $c$ of the conductor rings (Fig. $3 b, r=2.2$ $\mathrm{mm}$ and $s=0.25 \mathrm{~mm}$ fixed) or the slot $s$ between the rings (Fig. $3 c, r=2.2 \mathrm{~mm}$ and $c=0.3 \mathrm{~mm}$ fixed) increases from 0.1 to 0.5 . In Fig. $3 b$, the element value $C_{0}$ is
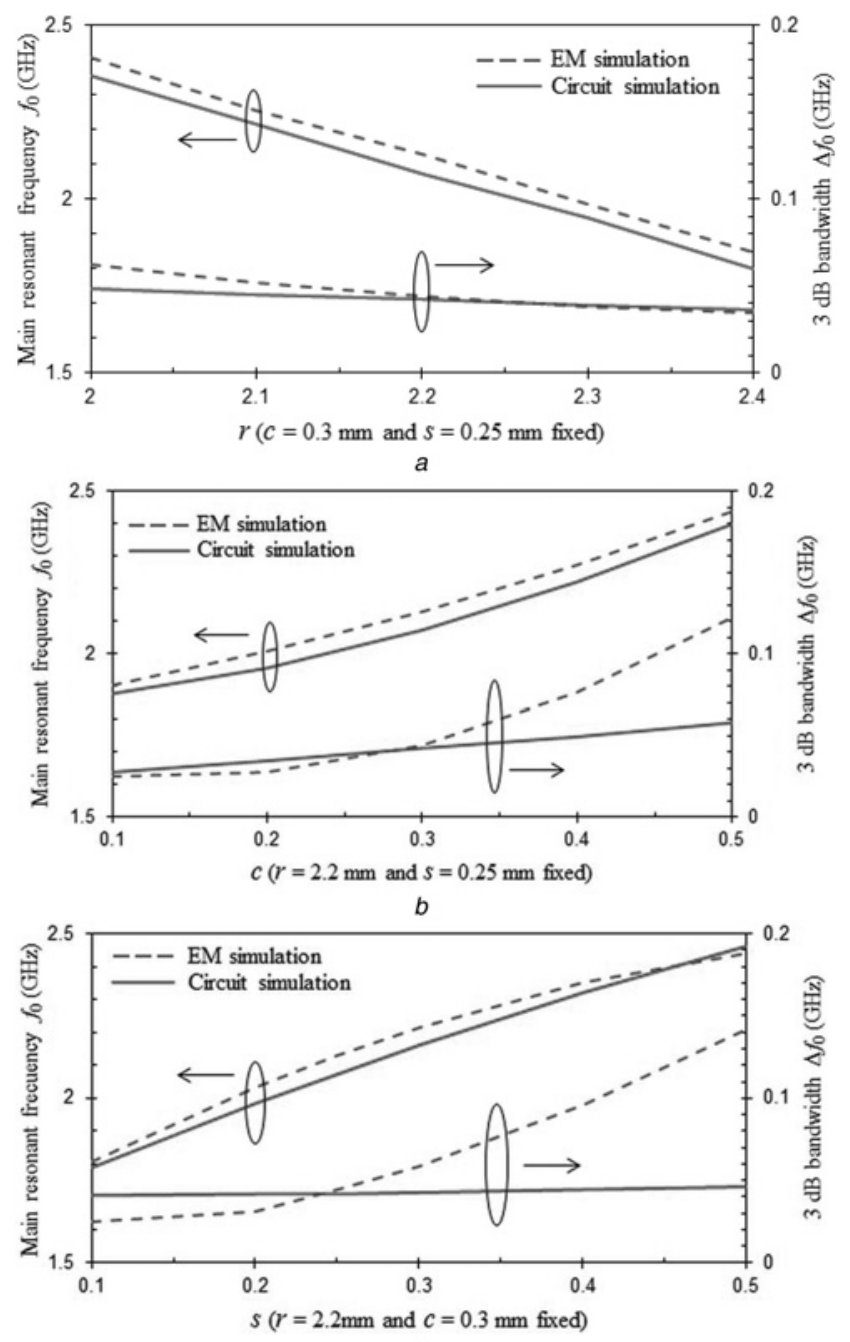

$c$

Fig. 3 Main resonant frequency $\left(f_{0}\right)$ and $3 d B$ stop-band bandwidth $\left(\Delta f_{0}\right)$ for the $C P W$ loaded with an OISRR as a function of the design parameters $r, c$ and $s$

$a$ Variation of $r$ ( $c=0.3 \mathrm{~mm}$ and $s=0.25 \mathrm{~mm}$ fixed)

$b$ Variation of $c(r=2.2 \mathrm{~mm}$ and $s=0.25 \mathrm{~mm}$ fixed $)$

$c$ Variation of $s$ ( $r=2.2 \mathrm{~mm}$ and $c=0.3 \mathrm{~mm}$ fixed)

quasi-constant because the design parameters $r$ and $s$ of the OISRR are fixed, while the other element value $L_{0}$ decreases as the width $c$ of the ring conductors increases. In Fig. $3 c$, the opposite occurs, the element value $L_{0}$ is quasi-constant because of the fixed design parameters $r$ and $c$ of the OISRR, while the other element value $C_{0}$ diminishes as the slot $s$ between the rings varies from 0.1 to 0.5 . Focusing on the EM simulations and according to the design parameters, $f_{0}$ is between 1.8 and $2.44 \mathrm{GHz}$ and $\Delta f_{0}$ varies between 0.025 and $0.15 \mathrm{GHz}$. The minimum and maximum relative errors between EM simulation and circuit results are, respectively, $0.77 \%$ and $2.7 \%$ for $f_{0}$ and $1.57 \%$ and $52.9 \%$ for $\Delta f_{0}$. The circuit model is less accurate for $\Delta f_{0}$ because of the variation of the impedance scaling factor $\gamma$, which is not taken into account in the circuit model. Considering this, the equivalent circuit model still provides a useful tool for the initial design of CPW notch filters.

\section{CPW notch filter using OISRR}

This proposed OISRR presents an interesting application in the design of compact notch filters. Thus, different 


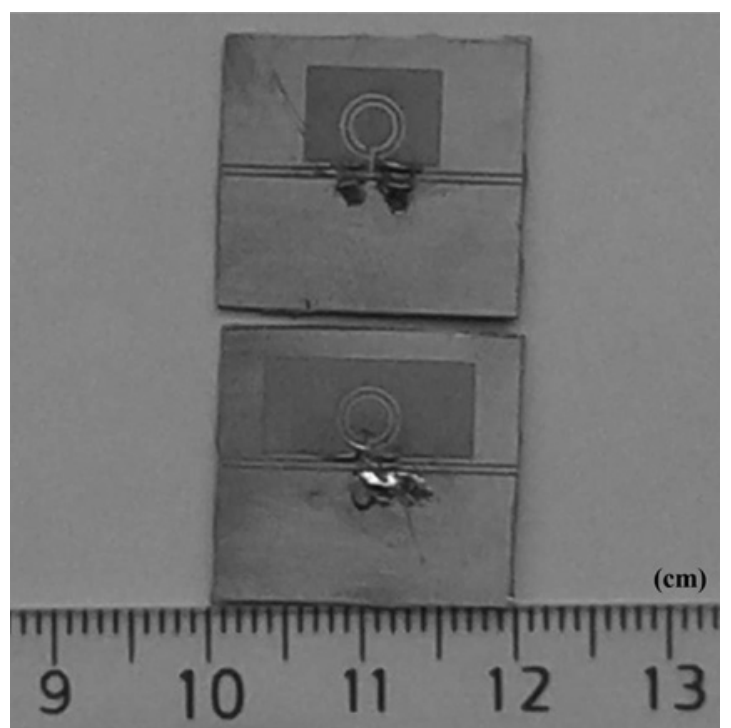

Fig. 4 Photographs of the fabricated CPW OISRR-based structures

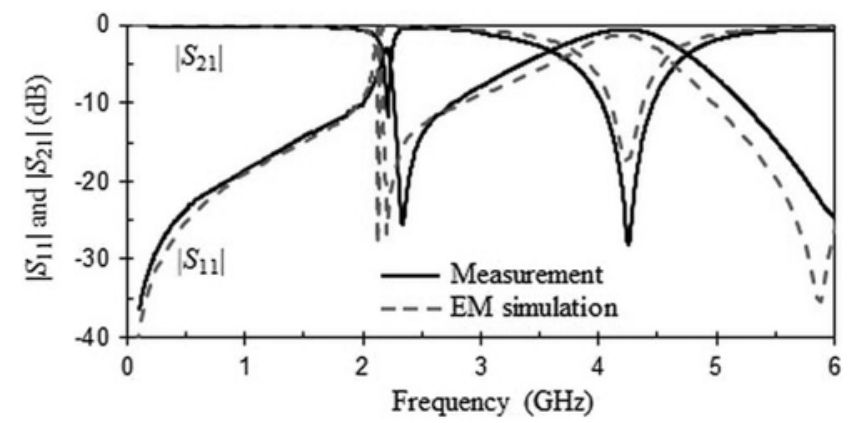

Fig. 5 Simulated and measured frequency responses for the $C P W$ loaded with an OISRR $\left(D_{I}=9 \mathrm{~mm}\right)$.

OISRR data: $r=2.2 \mathrm{~mm}, \quad c=0.3 \mathrm{~mm}, \quad s=0.25 \mathrm{~mm}$ and $D_{1} \times D_{2}=$ $9 \times 6.5 \mathrm{~mm}^{2}$

structures of the circuit shown in Fig. 1 have been fabricated (Fig. 4) on an Arlon $\mathrm{AD} 1000$ material $\left(\varepsilon_{r}=10.2, \operatorname{tg} \delta=\right.$ 0.0023 at $10 \mathrm{GHz}$, substrate thickness $h=0.635 \mathrm{~mm}$ and copper thickness $t=17.5 \mu \mathrm{m})$ and measured $(0.01-6 \mathrm{GHz})$ by means of a system of prototyping (LPKF protolaser S)

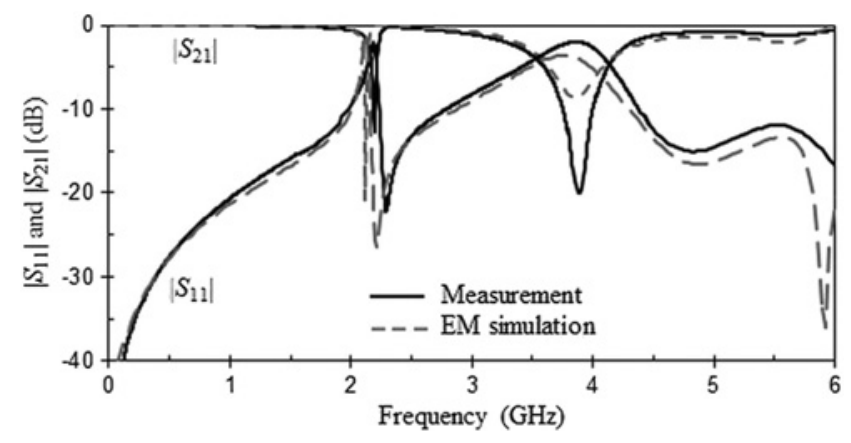

Fig. 6 Simulated and measured frequency responses for the $C P W$ loaded with an OISRR $\left(D_{1}=14 \mathrm{~mm}\right)$.

OISRR data: $r=2.2 \mathrm{~mm}, \quad c=0.3 \mathrm{~mm}, \quad s=0.25 \mathrm{~mm}$ and $D_{1} \times D_{2}=$ $14 \times 6.5 \mathrm{~mm}^{2}$ and a vector network analyser (Rohde \& Schwarz), respectively. The structures were connected to the vector network analyser by means of a commercial $50 \Omega$ coaxial to coplanar transition (Anritsu $3680 \mathrm{~K}$ test fixture). The system of measurement was calibrated from 0.01 to $6 \mathrm{GHz}$. The dimensions of the OISRR and CPW are the same as those used for the simulations shown in Fig. 2. Two different values of $D_{1}$ (length of etched windows, Fig. 1a) were used to evaluate its effect on the filter characteristics.

Figs. 5 and 6 show EM simulated and measured frequency responses of the CPW OISRR-based structures with $D_{1}=9$ and $14 \mathrm{~mm}$, respectively. As can be seen in these figures, the EM simulation and the measurement results present a reasonable agreement. The CPW OISRR-based filter with $D_{1}=9 \mathrm{~mm}$ (Fig. 5) has a main resonant frequency at $f_{0}=$ $2.2 \mathrm{GHz}$, an anti-resonance at $f_{\mathrm{a}}=2.33 \mathrm{GHz}$ and a spurious resonance at $f_{\mathrm{s}}=4.25 \mathrm{GHz}$, which is around two times the main resonant frequency $\left(f_{0}\right)$. The window $D_{1}$ only affects the spurious resonance. As $D_{1}$ is increased from two times (Fig. 5) to three times (Fig. 6) the diameter of the external ring $(\phi=2 r=4.4 \mathrm{~mm})$, the spurious resonance is shifted downwards of $0.37 \mathrm{GHz}\left(f_{\mathrm{s}}=3.88 \mathrm{GHz}\right)$ with a slight attenuation. However, the length $D_{1}$ has little effect on the main resonant frequency $\left(2.19 \leq f_{0}(\mathrm{GHz}) \leq 2.2\right)$, the $3 \mathrm{~dB}$ stop-band bandwidth at the main resonant frequency $\left(3.8 \leq \Delta f_{0}(\%) \leq 4.2\right)$ and the insertion loss in the main stop-band $(11.7 \leq I L(\mathrm{~dB}) \leq 12.5)$.

\section{Conclusion}

A novel compact notch filter in CPW technology based on an open interconnected ring resonator (OISRR) has been proposed and analysed. This OISRR is connected in parallel with a CPW and is modelled as a shunt series $L C$ resonant circuit. Simulation and measurement results have shown that this resonator works as a notch filter. The main resonant frequency of the OISRR is half the resonant frequency of the SRR with identical substrate and dimensions. With regards to a traditional CPW resonator with a length of $\lambda / 2$, the proposed CPW OISRR-based structure is approximately three times smaller. The designed filter presents a $3 \mathrm{~dB}$ stop-band bandwidth lower than $4.5 \%$ with more than $11 \mathrm{~dB}$ insertion loss. Thus, this resonator is expected to be useful in the design of compact CPW notch filters in microwave communication systems.

\section{Acknowledgment}

The authors gratefully acknowledge Ministerio de Ciencias e Innovación of Spain and FEDER for the financial support of this work under the grant no.: TEC2010-21520-C04-04/ TCM.

\section{References}

1 Hong, J.S., Lancaster, M.: 'Microstrip filters for RF/microwave applications' (John Wiley \& Sons, New York, 2001)

2 Durán-Sindreu, M., Vélez, A., Aznar, F., Sisó, G., Bonache, J., Martin, F.: 'Applications of open split ring resonators and open complementary split ring resonators to the synthesis of artificial transmission lines and microwave passive components', IEEE Trans. Microw. Theory Technol., 2009, 57, (12), pp. 3395-3403

3 Boutejdar, A., Elsherbini, A., Omar, S.: 'Method for widening the reject-band in low-pass/band-pass filters by employing coupled C-shaped defected ground structure', IET Microw. Antennas Propag., 2008, 2, (8), pp. 759-765 


\section{www.ietdl.org}

4 Ruiz, J.D., Martínez, F.L., Hinojosa, J.: '1D Koch fractal electromagnetic bandgap microstrip structures with $r / a$ ratios higher than 0.5', Microw. Opt. Technol. Lett., 2011, 53, (3), pp. 646-649

5 Ruiz, J.D., Martínez, F.L., Hinojosa, J.: 'Novel compact wide-band EBG structure based on tapered 1-D Koch fractal patterns', IEEE Antennas Wirel. Propag. Lett., 2011, 10, pp. 1104-1107

6 Martin, F., Facolne, F., Bonache, J., Marqués, R., Sorolla, M.: 'Miniaturized coplanar waveguide stop band filters based on multiple tuned split ring resonators', IEEE Microw. Wirel. Compon. Lett., 2003, 13, (12), pp. 511-513

7 Kim, J., Cho, C.S., Lee, J.W.: 'CPW bandstop filter using slot-type SRR', Electron. Lett., 2005, 41, (24), pp. 1333-1334

8 Vélez, A., Aznar, F., Durán-Sindreu, M., Bonache, J., Martín, F. 'Stop-band and band-pass filters in coplanar waveguide technology implemented by means of ellectrically small metamaterial-inspired open resonators', IET Microw. Antennas Propag., 2010, 4, (6), pp. 712-716
9 Al-Naib, I.A.I., Koch, M.: 'Highly miniaturised single metal layer CPW bandstop filters based on spiral resonators', Electron. Lett., 2010, 46, (18), pp. 1274-1275

10 Pendry, J.B., Holden, A.J., Ribbins, D.J., Stewart, W.J.: 'Magnetism from conductors and enhanced nonlinear phenomena', IEEE Trans. Microw. Theory Technol., 1999, 47, (11), pp. 2075-2084

11 Baena, J.D., Bonache, J., Martin, F., et al.: 'Equivalent-circuit models for split-ring resonators and complementary split-ring resonators coupled to planar transmission lines', IEEE Trans. Microw. Theory Technol., 2005, 53, (4), pp. 1451-1461

12 Martel, J., Marqués, R., Falcone, F., et al.: 'A new LC series element for compact bandpass filter design', IEEE Microw. Wirel. Compon. Lett., 2004, 14, (5), pp. 210-212

13 Koschny, T., Markos, P., Smith, D.R., Soukoulis, C.M.: 'Resonant and anti-resonant frequency dependence of the effective parameters of metamaterials', Phys. Rev. E., 2003, 68, 656021-656024 